(C) by R. Oldenbourg Verlag, München 1997

\title{
Crystal structure of two modifications of ammonium trifluoro nickelate(II), $\mathbf{N H}_{4} \mathrm{NiF}_{3}$
}

\section{Plitzko, M. Strecker}

Universität Hannover, Institut für Anorganische Chemie. Callinstr. 9. D-30167 Hannover, Germany

and G. Meyer

Universität Köln. Institut für Anorganische Chemie. Greinstr. 6. D-50939 Köln, Germany

Received June 17, 1996, transferred to 2nd update of database ICSD in 1997, CSD-No. 402540 and 402541

1. Crystal structure of the cubic low temperature modification of ammonium nickel trifluoride, $\mathrm{NH}_{4} \mathrm{NiF}_{3}-\mathrm{I}$

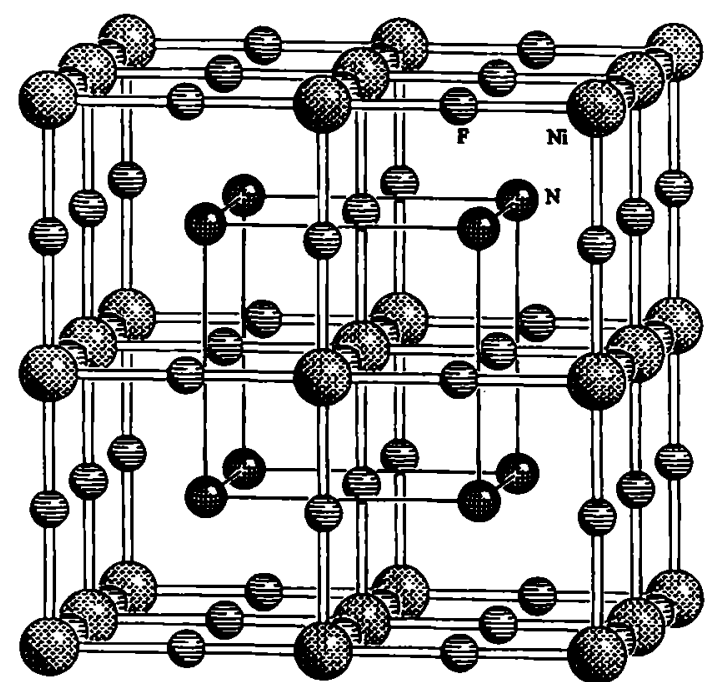

Source of material: $\mathrm{NH}_{4} \mathrm{NiF}_{3}-\mathrm{I}$ in the cubic (low temperature) modification was obtained from nickel powder and $\mathrm{NH}_{4} \mathrm{HF}_{2}$ (molar ratio 1:1.5) at $573 \mathrm{~K}$ (four weeks) in a sealed Monel metal container. The yellow powder contains many square yellow plates (see ref. 1).
$\mathrm{NH}_{4} \mathrm{NiF}_{3}$-I crystallizes like $\mathrm{KNiF}_{3}$ (see ref. 2) in the cubic $\mathrm{CaTiO}_{3}$ type of structure. The $\left[\mathrm{NiF}_{6}\right]$ octahedra share common corners with $\mathrm{Ni}^{2+}-\mathrm{F}$ distances of $204.38(14) \mathrm{pm}$ to form a three-dimensional network. The $\mathrm{Ni}^{2+}-\mathrm{Ni}^{2+}$ distances are $408.8(3) \mathrm{pm}$. $\mathrm{H}$ positions of $\mathrm{NH}_{4}{ }^{+}$are not determined.

$\mathrm{F}_{3} \mathrm{H}_{4} \mathrm{NN}$, cubic, $P m 3 m$ (No. 221), $a=4.088(3) \AA, V=68.3 \AA^{3}$, $Z=1, R(F)=0.026, R_{W}\left(F^{2}\right)=0.045$.

Table 1. Parameters used for the $\mathrm{X}$-ray data collection

$\begin{array}{ll}\text { Crystal: } & \text { yellow, square plate, size } 0.05 \times 0.20 \times 0.20 \mathrm{~mm} \\ \text { Wavelength: } & \text { Mo } K_{\alpha} \text { radiation }(0.71073 \AA) \\ \mu: & 67.10 \mathrm{~cm}^{-1} \\ \text { Diffractometer: } & \text { STOE IPDS } \\ \text { Scan mode: } & 20 \text { plates, } \Delta \varphi=5^{\circ} \\ \text { T }_{\text {measurement: }} & 293 \mathrm{~K} \\ 2 \theta_{\text {max }}: & 55.84^{\circ} \\ \text { N(hkl) } & 32 \\ \text { Criterion for } I_{0}: & I_{0}>2 \sigma\left(I_{0}\right) \\ \text { N(param) } & 5 \\ \text { Program: } & S H E L X L-93\end{array}$

Table 2. Final atomic coordinates and displacement parameters (in $\AA^{2}$ )

\begin{tabular}{|c|c|c|c|c|c|c|c|c|c|c|}
\hline Atom & Site & $x$ & $y$ & $z$ & $U_{11}$ & $U_{22}$ & $U_{33}$ & $U_{12}$ & $U_{13}$ & $U_{23}$ \\
\hline $\mathrm{Ni}$ & $1 b$ & $1 / 2$ & $1 / 2$ & $1 / 2$ & $0.0123(7)$ & $U_{11}$ & $U_{11}$ & 0 & 0 & 0 \\
\hline$F$ & $3 c$ & $1 / 2$ & $1 / 2$ & 0 & $0.018(2)$ & $U_{11}$ & $0.013(3)$ & 0 & 0 & 0 \\
\hline $\mathbf{N}$ & la & 0 & 0 & 0 & $0.017(3)$ & $U_{11}$ & $U_{11}$ & 0 & 0 & 0 \\
\hline
\end{tabular}




\section{Crystal structure of the hexagonal high temperature modification of ammonium nickel trifluoride, $\mathrm{NH}_{4} \mathrm{NiF}_{3}$-II}

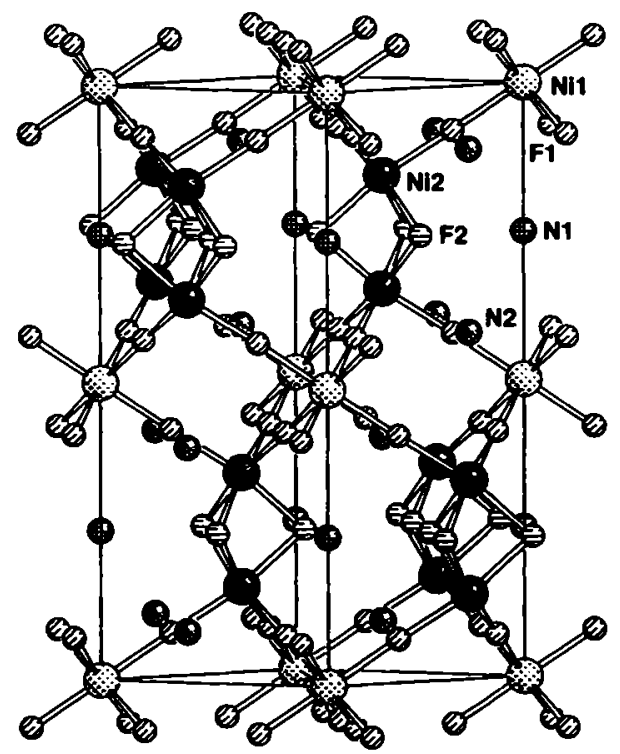

Source of material: $\mathrm{NH}_{4} \mathrm{NiF}_{3}-\mathrm{II}$ in the hexagonal (high temperature) modification was obtained from nickel powder and $\mathrm{NH}_{4} \mathrm{HF}_{2}$ (molar ratio 1:2) at $673 \mathrm{~K}$ (four weeks) in a sealed Monel metal container in the presence of a tungsten sheet on which the green crystals grew (see ref. 3).
$\mathrm{NH}_{4} \mathrm{NiF}_{3}$-II crystallizes like $\mathrm{RbNiF}_{3}$ (see ref. 4) in the hexagonal $\mathrm{BaTiO}_{3}$ type of structure. Two $\left[\mathrm{NiF}_{6}\right]$ octahedra share a common face forming a double octahedron $\left[\mathrm{Ni}_{2} \mathrm{~F} 9\right]$ with $\mathrm{Ni}^{2+}-\mathrm{F}(2)^{-}$distances of 201.1(2) pm (6x) and $\mathrm{Ni}^{2+}-\mathrm{F}(1)^{-}$distances of 203.8(2) pm (6x). These double octahedra share common comers with $\left[\mathrm{NiF}_{6}\right]$ octahedra to form a three-dimensional network. The $\mathrm{Ni}^{2+}-\mathrm{F}(1)^{-}$distances are 202.6(1) pm (6x) in these ideal octahedra. The $\mathrm{Ni}^{2+}-\mathrm{Ni}^{2+}$ distance in the confacial bioctahedron is $275.0(1) \mathrm{pm}$. The $\mathrm{H}$ postions are not determined.

$\mathrm{F}_{3} \mathrm{H}_{4} \mathrm{NNi}$, hexagonal, $P 63 / m m c$ (No. 194), $a=5.8456(3) \AA$, $c=14.3563(3) \AA, V=424.9 \AA^{3}, Z=6, R(F)=0.024, R_{w}\left(F^{2}\right)=0.083$.

Table 3. Parameters used for the $\mathrm{X}$-ray data collection

$\begin{array}{ll}\text { Crystal: } & \text { geen, rectangular plate, size } 0.05 \times 0.20 \times 0.10 \\ & \mathrm{~mm} \\ \text { Wavelength: } & \text { Mo } K_{\alpha} \text { radiation }(0.71073 \AA) \\ \mu: & 66.48 \mathrm{~cm}^{-1} \\ \text { Diffractometer: } & \text { Siemens-Stoe } \\ \text { Scan mode: } & \text { profile fitted } \omega / 2 \theta \\ T_{\text {measurement: }} & 293 \mathrm{~K} \\ 2 \theta_{\text {max }} & 80.1^{\circ} \\ N(\text { hkl) })_{\text {unique: }} & 554 \\ \text { Criterion for } I_{0}: & I_{0}>2 \sigma\left(I_{0}\right) \\ \text { N(param) } & 21 \\ \text { Program: } & S H E L X L-93\end{array}$

Table 4. Final atomic coordinates and displacement parameters (in $\AA^{2}$ )

\begin{tabular}{lllllllllll}
\hline Atom & Site & $x$ & $y$ & $z$ & $U_{11}$ & $U_{22}$ & $U_{33}$ & $U_{12}$ & $U_{13}$ & $U_{23}$ \\
\hline $\mathrm{Ni}(1)$ & $2 a$ & 0 & 0 & 0 & $0.0127(2)$ & $U_{11}$ & $0.0132(3)$ & $U_{11} / 2$ & 0 & 0 \\
$\mathrm{Ni(2)}$ & $4 f$ & $1 / 3$ & $2 / 3$ & $0.84581(3)$ & $0.0135(1)$ & $U_{11}$ & $0.0129(2)$ & $U_{11} / 2$ & 0 & 0 \\
$\mathrm{~F}(1)$ & $6 h$ & $0.5181(2)$ & $2 x$ & $1 / 4$ & $0.0210(7)$ & $0.0154(8)$ & $0.0163(7)$ & $U_{22} / 2$ & 0 & 0 \\
$\mathrm{~F}(2)$ & $12 k$ & $0.8342(2)$ & $2 x$ & $0.07898(9)$ & $0.0216(5)$ & $0.0170(6)$ & $0.0195(6)$ & $U_{22} / 2$ & $0.0028(2)$ & $2 U_{13}$ \\
$\mathrm{~N}(1)$ & $2 b$ & 0 & 0 & $1 / 4$ & $0.018(1)$ & $U_{11}$ & $0.019(2)$ & $U_{11} / 2$ & 0 & 0 \\
$\mathrm{~N}(2)$ & $4 f$ & $1 / 3$ & $2 / 3$ & $0.0953(3)$ & $0.0188(8)$ & $U_{11}$ & $0.019(1)$ & $U_{11} / 2$ & 0 & 0 \\
\hline
\end{tabular}

Acknowledgment. We thank the Deutsche Forschungsgemeinschaft for financial support.

\section{References}

1. Plitzko, C.: Neue komplexe Ammoniumfluoride und Fluorid-Ammoniakate durch Umsetzung von Metallen mit Ammonium- und Hydraziniumfluoriden. Dissertation, University of Hannover, Germany 1996.
2. Lule, A.; Schmitz-DuMont, O.: Struktur und Remissionsspektren der Ammoniumfluoroniccolate. Z. Anorg. Allg. Chem. 389 (1972) 1-10.

3. Strecker, M.: Synthese von Fluoriden mit Ammoniumfluoriden und Polytetrafluorethylen. Dissertation, University of Hannover, Germany 1994.

4. Babel, D.: Die Kristallstrukturen der hexagonalen Fluoroperowskite. Z. Anorg. Allg. Chem. 369 (1969) 117-130.

5. Sheldrick, G. M.: SHELXL-93, a program for refining crystal structures. University of Göttingen, Germany 1993. 